\title{
Linfoma Primário de Cólon: Relato de Caso
}

\author{
Primary Colorectal Lymphoma: Case Report
}

\author{
RAFAELLUÍS LUPORINI'; ${ }^{1}$ ANTONIO CARLOS ROMAJÚNIOR²; ELAINE CRISTINA HENRIQUEALMEIDA; \\ MARCELO RODOLFO MARCIANO ${ }^{4}$; LUIZ VAGNER SIPRIANI ${ }^{5}$; FRANCISCO DEASSIS GONÇALVES FILHO ${ }^{6}$;

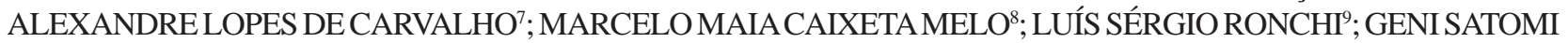 \\ CUNRATH $^{10}$; JOÃO GOMES NETINHO ${ }^{11}$
}

\begin{abstract}
1. Médico residente em Coloproctologia - Faculdade de Medicina de São José do Rio Preto (FAMERP) / Disciplina de Coloproctologia FSBCP; ${ }^{2}$ Médico Cirurgião Geral - Faculdade de Medicina de São José do Rio Preto (FAMERP);

3. Médica Coloproctologista - Faculdade de Medicina de São José do Rio Preto (FAMERP) / Disciplina de Coloproctologia FSBCP; ${ }^{4}$. Médico residente em Coloproctologia - Faculdade de Medicina de São José do Rio Preto (FAMERP) / Disciplina de Coloproctologia HB-FAMERP, São José do Rio Preto, SP, FSBCP; ${ }^{5}$. Médico residente em Coloproctologia - Faculdade de Medicina de São José do Rio Preto (FAMERP) / Disciplina de Coloproctologia FSBCP; ${ }^{6}$ Médico Coloproctologista - Faculdade de Medicina de São José do Rio Preto (FAMERP) / Disciplina de Coloproctologia Titulo de especialista pela SBCP / ASBCP; ${ }^{7}$. Médico Coloproctologista - Faculdade de Medicina de São José do Rio Preto (FAMERP) / Disciplina de Coloproctologia. Titulo de especialista pela SBCP / ASBCP; ${ }^{8}$ Médico

Coloproctologista - Faculdade de Medicina de São José do Rio Preto (FAMERP) / Disciplina de Coloproctologia.

Titulo de especialista pela SBCP e CBCD / ASBCP. Mestre em ciências da saúde pela FAMERP. Médico da disciplina de coloproctologia da FAMERP; 9. Faculdade de Medicina de São José do Rio Preto (FAMERP) / Disciplina de

Coloproctologia. Título de especialista pela SBCP e CBCD / ASBCP. Médico contratado da disciplina de coloproctologia da FAMERP; ${ }^{10}$ Médico Coloproctologista - Faculdade de Medicina de São José do Rio Preto (FAMERP) / Disciplina de Coloproctologia. Título de especialista pela SBCP e CBCD / TSBCP. Mestre em ciências da saúde pela FAMERP. Sub-chefe da disciplina de coloproctologia da FAMERP; ${ }^{11}$. Médico Coloproctologista - Faculdade de Medicina de São José do Rio Preto (FAMERP) / Disciplina de ColoproctologiaTítulo de Especialista SBCP e AMB /

TSBCP. Mestre em Ciencias da Saúde FAMERP. Doutor em Cirurgia UNICAMP. Chefe da Disciplina de Coloproctologia HB / FAMERP. Vice Mestre do CBC Regional Noroeste do ESP.
\end{abstract}

LUPORINI RL; ROMA JÚNIOR AC; ALMEIDA ECH; MARCIANO MR; SIPRIANI LV; GONÇALVES FILHO FA; CARVALHO AL; MELO MMC; RONCHI LS; CUNRATH GS; NETINHO JG. Linfoma Primário de Cólon: Relato de Caso. Rev bras Coloproct, 2010;30(3): 356-359.

RESUMO: O linfoma colorretal primário é uma doença rara (0.2 a $0.6 \%$ de todas as neoplasias colônicas), apresentando pior prognóstico quando comparado com o linfoma gástrico primário ou com o adenocarcinoma do cólon. É uma doença com sintomatologia inespecífica, o que dificulta o diagnóstico precoce. O objetivo deste relato é mostrar um caso de linfoma primário do cólon, revisar critérios diagnósticos e tratamento.

Descritores: Linfoma colônico primário, neoplasia colônica, linfoma gastrointestinal.

\section{INTRODUÇÃO}

Os linfomas normalmente apresentam-se de forma disseminada, podendo, no entanto aparecer no trato gastrointestinal em formas localizadas, principal- mente no estômago e intestino delgado, sendo os linfomas primários do cólon raros $^{1,2}$. Apresentam sistemas de classificação e diagnósticos diferentes do adenocarcinoma de cólon ${ }^{3}$, possuindo também desfecho clínico mais grave do que esta patologia ${ }^{2}$, sendo

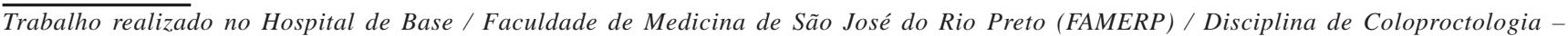
Departamento de Cirurgia
}

Recebido em 22/09/2009

Aceito para publicação em 12/11/2009 
necessário um alto nível de suspeição clínica para a realização de um diagnóstico precoce. O correto estadiamento permite predizer o prognóstico e direcionar a terapêutica, sendo a chance de cura, uma cirurgia para ressecção tumoral em estadios preco$\operatorname{ces}^{2,3,4}$.

\section{RELATO DE CASO}

FPB, 65 anos, sexo feminino, admitida com queixa de dor em fossa ilíaca direita e abaulamento local há 2 meses, associado a hiporexia, constipação e emagrecimento de $5 \mathrm{~kg}$ no período. Há 4 dias da admissão referia febre diária não aferida, sendo encontrada ao exame físico massa endurecida e fixa em fossa ilíaca direita.

Realizada tomografia computadorizada de abdômen que demonstrou espessamento concêntrico do cólon ascendente, com imagem irregular, heterogênea, hipodensa, entremeada por áreas hipoatenuantes e com gás em seu interior.

A colonoscopia evidenciou lesão ulcerada de bordas elevadas ocupando toda luz cólica direita, sendo realizada biópsia que evidenciou colite crônica ulcerativa inespecífica.

Paciente foi submetida a laparotomia exploradora dada a lesão sugerir tratar-se de neoplasia sendo identificada massa em cólon ascendente. Realizada ressecção em bloco do transverso proximal, cólon ascendente, ceco (figura 1), $30 \mathrm{~cm}$ de íleo terminal e parte da parede pélvica com confecção de

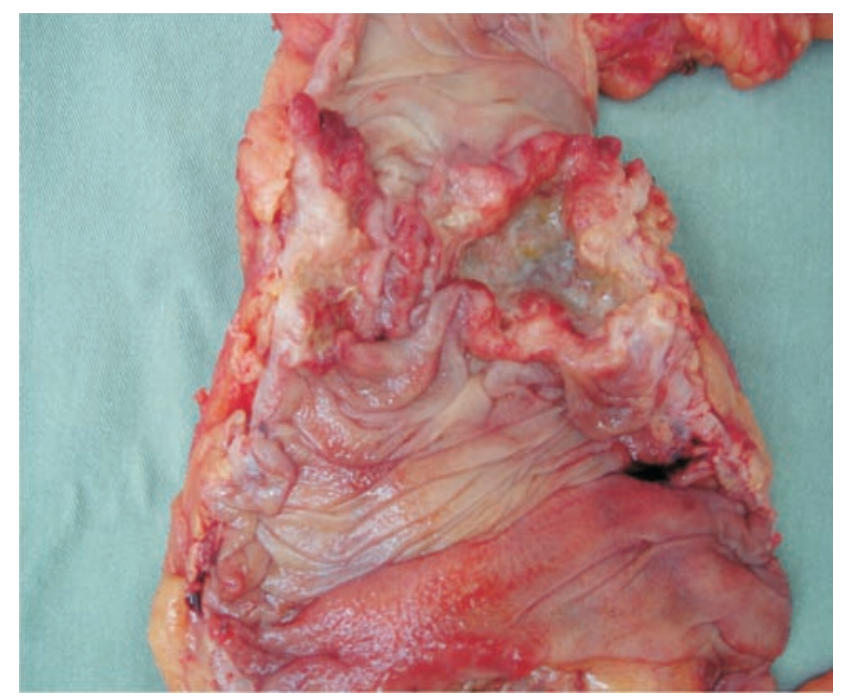

Figura 1 - Peça de Ressecção Cirúrgica. ileotransversoanastomose. Paciente recebeu alta no $3^{\circ}$ pós-operatório.

O exame anátomo-patológico da peça demonstrou neoplasia maligna pouco diferenciada, sugestiva de linfoma. (figura 2). Realizada imunohistoquimímica que demonstrou linfoma difuso de grandes células (WHO 2001), revelando imunofenótipo B (CD 20+), variante morfológica anaplásica e índice de proliferação celular de 70\%. Estadio I de Ann Arbor modificado por Mussheff and Schimidt Volmer.

Paciente apresentou boa evolução, sendo submetida a tratamento quimioterápico com esquema RCHOP (rituximabe, ciclofosfamida, doxorrubicina, vincristina e prednisolona), estando assintomática e sem evidências de recidiva em dois anos de acompanhamento.

\section{DISCUSSÃO}

Os linfomas primários do cólon correspondem a 0,2 a $0,6 \%$ de todas as neoplasias cólicas, sendo seu pico de incidência entre 50 e 70 anos $^{1}$. É mais predominante em homens, tendo igual incidência entre os sexos quando se considera apenas adultos ${ }^{2}$. São lesões mais comuns no ceco, fato talvez explicado pela maior quantidade de linfonodos neste local ${ }^{3}$. A maioria trata-se de uma lesão única. Os fatores de risco são: colite ulcerativa, doença de Chron, radioterapia prévia, transplante renal, ureterossigmoidostomia, corticoterapia e HIV (o que pode explicar o aumento na incidência) $)^{3}$.

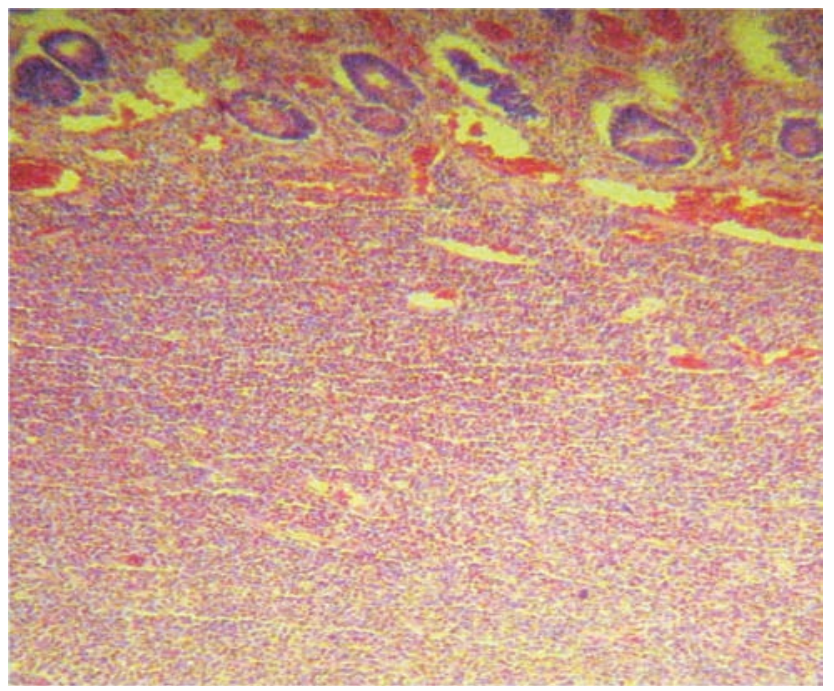

Figura 2 - Microscopia. 
Tabela 1 - Critérios de Dawnson para linfoma primário.

1 - Ausência de linfonodos superficiais palpáveis no primeiro exame

2 - Radiografia de Tórax normal

3 - Verificar à laparotomia comprometimento exclusivo do segmento gastrointestinal e dos seus linfonodos regionais

4 - Leucometria total e diferencial normal

5 - Ausências de lesões hepatoesplênicas

6 - Ausências de linfonodomegalias detectáveis à Tomografia computadorizada

7 - Normalidades das células da medula óssea

Tabela 2 - Classificação de Ann Arbor Modificada.

\begin{tabular}{ll}
\hline Estadio I & Tumor confinado ao trato gastrointestinal (IE) \\
Estadio II & $\begin{array}{l}\text { Tumor com envolvimento linfonodal regional (IIE 1) } \\
\text { Envolvimento linfonodal além dos linfonodos regionais (IIE 2) }\end{array}$ \\
Estadio III & Tumor com outros envolvimentos linfonodais regionais(fígado e baço) ou órgãos extra-abdominais \\
\hline
\end{tabular}

Os sintomas mais comuns são dor abdominal crônica inespecífica, perda de peso, massa palpável e alteração do hábito intestinal, (semelhantes ao apresentados inicialmente pela paciente), podendo também apresentar-se com náuseas, vômitos, febre, sangramento gastrointestinal ou abdome agudo ${ }^{1,2,3,4}$. Os critérios de Dawson são usados para o diagnósti$\mathrm{co}^{2}$. (tabela 1)

Os exames de imagem ajudam o diagnóstico e no estadiamento, no entanto o diagnóstico definitivo é feito apenas através da análise da biópsia, podendo esta ser feita por colonoscopia ou por análise histológica da peça obtida na ressecção cirúrgica ${ }^{2}$. No caso apresentado a biópsia realizada pela colonoscopia mostrou apenas a reação inflamatória no cólon.

A maioria dos linfomas de cólon são provenientes das células $\mathrm{B}^{2,3,4}$, sendo este o tipo encontrado no caso apresentado. Para o estadiamento o sistema mais usado é o de Ann Arbor, inicialmente proposto para estadiamento dos linfomas de Hodgkin. Foi modificado por Mussheff and Schimidt Volmer para ser mais bem aplicado aos linfomas gastrointestinais ${ }^{3,5}$. (tabela 2)

O tratamento é eminentemente cirúrgico, podendo ser realizado tanto por via aberta ou laparoscópica ${ }^{2}$, com taxas de morbidade e mortalidade menores neste. O uso da quimioterapia adjuvante é
Tabela 3 - Critérios de Pior Prognóstico.

\begin{tabular}{l}
1 - Envolvimento linfonodal regional \\
2 - Tumores maiores do que $5 \mathrm{~cm}$ \\
3 - Ressecções tumorais incompletas \\
$4-$ Tumores perfurados \\
$5-$ Tumores com pouca diferenciação histológica \\
\hline
\end{tabular}

controverso $^{1,4,5,6,7}$, sendo que alguns autores a usam em todos os casos e outros apenas em tumores de pior prognóstico (tabela 3), podendo-se também usar a radioterapia em casos de linfoma de reto ${ }^{1,2}$.

\section{CONCLUSÃO}

Embora raro, o linfoma primário de cólon é uma patologia que merece todo o cuidado, pois é mais agressivo que o tumor de cólon ${ }^{2}$ e a sua cura depende principalmente de um diagnóstico nos estádios precoces, pois o melhor tratamento é a ressecção radical ${ }^{2,3,4}$, sendo isto difícil devido aos sintomas pouco específicos no início da doença. A quimioterapia adjuvante vem demonstrando bons resultados ${ }^{3,5,7}$. Devido à raridade do caso se faz necessário a descrição de relatos para conhecimento médico dos aspectos clínicos, diagnósticos e terapêuticos da patologia. 
ABSTRACT: The primary colorectal lymphoma is a rare disease ( 0.2 to $0.6 \%$ of all colonic neoplasias), that has a worse prognosis than primary gastric lymphoma or colon adenocarcinoma. The poor signals makes the early diagnosis difficult. The objectives of this report is to describe a case of primary colon lymphoma, revise diagnosis criteria and treatment.

Key words: Primary colonic lymphoma, colon neoplasia, gastrointestinal lymphoma.

\section{REFERÊNCIAS}

1 - Wong MT, Eu KW. Primary colorectal lymphomas.Colorectal Dis. 2006; 8(7): 586-591.

2 - Waisberg J, Bromberg SH, Santos HVB, Barretto E, Giordano FC \& Godoy AC. Linfoma primário perfurado de cólon. Relato de caso. Aspectos clínicos-patológicos e estudo imunohistoquímico. Rev Bras ColoProct. 1994; 14(3): 175-178.

3 - Martínez-Ramos D, Gibert-Gerez J, Manuel Miralles-Tena JM, Martínez-Banaclocha M, Escrig-Sos J, Salvador-Sanchís JL. Laparoscopic colectomy for primary colonic lymphoma. Rev Esp Enferm Dig. 2005; 97: 744-749.

4 - Doolabh N, Anthony T, Simmang C, Bieligk S, Lee E, Huber P, Hughes R, Turnage R - Primary colonic lymphoma. J Surg Oncol. 2000; 74(4):257-62

5 - Waisberg J, Bromberg SH, Franco MI, Matheus CO, Zanotto A, Petrolino LF, ET AL. Primary non-Hodgkin lymphoma of the right colon: a retrospective clinical-pathological study. Int Surg. 2001; 86(1): 20-25.

6 - Gonzales QH, Heslin MJ, Dávila-Cervantes A, Alvarez-Tostado J, de los Monteros AE, Shore G, ET AL. Primary colonic lymphoma. Am Surg. 2008; 74(3): 214-216.

7 - Fan CW, Changchien CR, Wang JY, Chen JS, Hsu KC, Tang R, Chiang JM. Primary Colorectal Lymphoma. Colorectal Dis. 2000; 43(9): 1277-1282.

Endereço para correspondência:

RAFAEL LUÍS LUPORINI

Rua 15 de Novembro, 3426

São Carlos - SP

CEP:13569-220

Telefone: (16) 3372-1183 / (17) 8111-5500

E-mail: rafaelluisluporini@yahoo.com.br 